Moroccan J. of Pure and Appl. Anal. (MJPAA)

Volume 7(1), 2021, Pages 80-87

ISSN: Online 2351-8227 - Print 2605-6364

DOI: $10.2478 / \mathrm{mjpaa}-2021-0008$

\title{
On the quasi-Fredholm and Saphar spectrum of strongly continuous Cosine operator function
}

HAMID BOUA $^{1}$

Aвstract. Let $(C(t))_{t \in \mathbb{R}}$ be a strongly continuous cosine family and $A$ be its infinitesimal generator. In this work, we prove that, if $C(t)-\cosh \lambda t$ is Saphar (resp. quasi-Fredholm) operator and $\lambda t \notin i \pi \mathbb{Z}$, then $A-\lambda^{2}$ is also Saphar (resp. quasi-Fredholm) operator. We show by counter-example that the converse is false in general.

Mathematics Subject Classification (2010). 47A10, 47D62.

Key words and phrases. Cosine, Quasi-Fredholm, Semi-regular, Saphar.

\section{Introduction}

Let $X$ be a complex Banach space, $\mathcal{B}(X)$ denotes the algebra of all bounded linear operators on $X$ and $\mathcal{C}(X)$ the set of all linear closed operators from $X$ to $X$. We write $\mathcal{D}(T), \mathcal{R}(T)$, $\mathcal{R}^{\infty}(A)=\cap_{n \geq 0} \mathcal{R}\left(A^{n}\right), \mathcal{N}(T), \rho(T), \sigma(T), \sigma_{p}(T)$ and $\sigma_{r}(T)$ respectively for the domain, the range, the hyper-range, the kernel, the resolvent, the spectrum, the point spectrum and residual spectrum of an operator $T \in \mathcal{C}(X)$. The function resolvent of $T \in \mathcal{C}(X)$ is defined for all $\lambda \in \rho(T)$ by $R(\lambda, T)=(\lambda-T)^{-1}$.

Recall that a closed operator $T$ is said to be a semi-regular operator or semi-regular if $\mathcal{R}(T)$ is closed and $\mathcal{N}(T) \subseteq \mathcal{R}^{\infty}(T)$, see [6, Theorem 2.10].

Received 08 August 2020 - Accepted: 08 November 2020.

(C) The Author(s) 2020. This article is published with open access by Sidi Mohamed Ben Abdallah University.

${ }^{1}$ Department of Mathematics and Computer Science, Pluridisciplinary Faculty of Nador, Mohammed First University. e-mail: h.boua@ump.ac.ma. 
A closed operator $S$ is called a generalized inverse of $T$ if $\mathcal{R}(T) \subseteq \mathcal{D}(S), \mathcal{R}(S) \subseteq \mathcal{D}(T)$, $T S T=T$ on $\mathcal{D}(T)$ and $S T S=S$ on $\mathcal{D}(S)$, see [3, Definition 1.1].

A closed operator $T$ is called a Saphar operator if $T$ has a generalized inverse and $\mathcal{N}(T) \subseteq$ $\mathcal{R}^{\infty}(T)$.

Let $T \in \mathcal{C}(X)$ and let $\Delta(T)=\left\{n \in \mathbb{N}: \forall m \geq n, \mathcal{R}\left(T^{n}\right) \cap \mathcal{N}(T)=\mathcal{R}\left(T^{m}\right) \cap \mathcal{N}(T)\right\}$. The degree of stable iteration $\operatorname{dis}(T)$ of $T$ is defined as $\operatorname{dis}(T)=\inf \Delta(T)$ with $\operatorname{dis}(T)=\infty$ if $\Delta(T)=\varnothing$, see [4]. $T$ is called a quasi-Fredholm operator of degree $d$ if there exists an integer $d \in \mathbb{N}$ such that

(1) $\operatorname{dis}(T)=d$;

(2) $\mathcal{R}\left(T^{n}\right)$ is closed in $X$ for all $n \geq d$;

(3) $\mathcal{R}(T)+\mathcal{N}\left(T^{n}\right)$ is closed in $X$ for all $n \geq d$.

The semi-regular, Saphar and quasi-Fredholm spectra are defined by:

$$
\begin{gathered}
\sigma_{K}(T)=\{\lambda \in \mathbb{C}: T-\lambda \text { is not semi-regulier }\}, \\
\sigma_{\text {Sap }}(T)=\{\lambda \in \mathbb{C}: T-\lambda \text { is not Saphar }\}, \\
\sigma_{q F}(T)=\{\lambda \in \mathbb{C}: T-\lambda I \text { is not quasi-Fredholm }\} .
\end{gathered}
$$

Consider in $X$ the well-posed Cauchy problem

$$
(P)\left\{\begin{array}{l}
u^{\prime \prime}(t)=A u(t), \quad t \in \mathbb{R} \\
u(0)=u_{0} \\
u^{\prime}(0)=u_{1} .
\end{array}\right.
$$

Where $A: X \longrightarrow X$ is a densely defined closed operator with nonempty resolvent set $\rho(A)$. The problem $(P)$ is (see [2], [5] and [10]) well-posed if and only if $A$ generates a strongly continuous cosine operator function $(C(t))_{t \in \mathbb{R}}$, i.e., a family of operators satisfying the following conditions:

(1) $C(t+s)+C(t-s)=2 C(t) C(s)$ for all $t, s \in \mathbb{R}$.

(2) $C(0)=I$ (the identity operator).

(3) $t \rightarrow C(t)$ is continuous on $\mathbb{R}$ with respect to the operator norm topology on $\mathcal{B}(X)$.

There exist some $M \geq 1, \omega \in \mathbb{R}$ such that $\|C(t)\| \leq M e^{\omega t}$ for all $t \geq 0$.

If $(C(t))_{t \in \mathbb{R}}$ is a strongly continuous cosine operator function, then the infinitesimal generating operator $A$ is defined by

$$
D(A)=\left\{x \in X: \lim _{s \rightarrow 0} \frac{2(C(s) x-x)}{s^{2}} \text { exists }\right\}
$$

and

$$
A x=\lim _{s \rightarrow 0} \frac{2(C(s) x-x)}{s^{2}}=C^{\prime \prime}(0) .
$$

A solution of problem $(P)$ is given with the help of a strongly continuous cosine operator function by the formula $u(t)=C(t) u_{0}+S(t) u_{1}$ for $t \in \mathbb{R}$, where $S(t)$ is the sine operator function associated with the $(C(t))_{t \in \mathbb{R}}$ and is defined as $S(t) x:=\int_{0}^{t} C(s) x d s, t \in \mathbb{R}, x \in X$. In this work we will use the theory of integration in the sense of Bochner. 
If $(C(t))_{t \in \mathbb{R}}$ is a uniformly continuous operator cosine function then there is an $A \in \mathcal{B}(X)$ with $C(t)=\cosh t \sqrt{A}, t \in \mathbb{R}$. For $t \in \mathbb{R}$, the function $f: z \in \mathbb{C} \mapsto \cosh t \sqrt{z}$ defines an entire function. Thus, according to the spectral mapping theorem, we have $\cosh t \sqrt{\sigma_{\star}(A)}=$ $\sigma_{\star}(C(t))$, for all $t \in \mathbb{R}$, with $\sigma_{\star}$ the spectrum corresponding to regularity in the sense of $\mathrm{V}$. Müller [7, Definition 6.1].

The following spectral inclusion $\cosh t \sqrt{\sigma(A)} \subseteq \sigma(C(t)), t \in \mathbb{R}$ was obtained by B. Nagy; he also gave an example where the reverse inclusion fails [8] and he showed that $\sigma_{\star}(C(t))=$ $\cosh t \sqrt{\sigma_{\star}(A)}, t \in \mathbb{R}$, with $\star \in\{p, r\}$.

However there are several large classes of generators $A$ for which the spectrum of $C(t)$ can be expressed in terms of $\sigma(A)$, namely $\sigma(C(t))=\overline{\cosh t \sqrt{\sigma(A)}}, t \in \mathbb{R}$, if $A$ is the generator of a uniformly bounded cosine function on a Hilbert space [11] or of a cosine function of normal operators [12].

In this paper, we continue to study the spectral theory of strongly continuous cosine operator function. we prove that, if $C(t)-\cosh \lambda t$ is Saphar (resp. quasi-Fredholm) operator and $\lambda t \notin i \pi \mathbb{Z}$, then $A-\lambda^{2}$ is also Saphar (resp. quasi-Fredholm) operator. We show by counterexample that the converse is false in general.

\section{Main results}

Now, the following theorem state the main result of this article.

Theorem 1. Let $(C(t))_{t \in \mathbb{R}}$ be a strongly continuous cosine operator function on $X$ with generator $A$. For all $t \neq 0$, we have,

(1) if $\lambda^{2} \in \sigma_{\text {Sap }}(A)$ and $\lambda t \notin i \pi \mathbb{Z}$, then $\cosh \lambda t \in \sigma_{\text {Sap }}(C(t))$,

(2) if $\lambda^{2} \in \sigma_{q F}(A)$ and $\lambda t \notin i \pi \mathbb{Z}$, then $\cosh \lambda t \in \sigma_{q F}(C(t))$.

Theorem 1 then directly yields the following important result:

Corollary 2. Let $A$ be the generator of a strongly continuous cosine operator function $(C(t))_{t \in \mathbb{R}}$. Then for all $t \neq 0$,

$$
\cosh t \sqrt{\sigma_{\star}(A)} \cup\{-1,1\} \subseteq \sigma_{\star}(C(t)) \cup\{-1,1\},
$$

with $\star \in\{$ Sap,$q F\}$.

Remark 3. Let $X$ be the complex $\ell^{2}$ space. For $\left(z_{n}\right)_{n \in \mathbb{N}} \in \ell^{2}$ and $s \in \mathbb{R}$, we put $C(s)\left(z_{n}\right)_{n}=$ $\left(\cos (n s) z_{n}\right)_{n}$. Then $A\left(z_{n}\right)_{n}=\left(-n^{2} z_{n}\right)_{n}$ with $\mathcal{D}(A)=\left\{\left(z_{n}\right)_{n} \in \ell^{2}: \sum_{n=1}^{\infty} n^{4}\left|z_{n}\right|^{2}<\infty\right\}$ and $\sigma(A)=\sigma_{p}(A)=\left\{-n^{2}: n \in \mathbb{N}^{*}\right\}$. Then cosh $\sqrt{\sigma_{\text {Sap }}(A)}$ is countable. From [8], $\sigma(C(1))$ contains the set $[-1,1] \backslash\left\{\cos n: n \in \mathbb{N}^{*}\right\}$ which is uncountable set. Then $\sigma(C(1))$ is uncountable. By $[9$, Corollary 2.10], we have $\sigma_{S a p}(C(1))$ and $\sigma_{q F}(C(1))$ are uncountable. This shows that all of the above inclusions are strict.

The following lemmas are the most widely used results of this paper. 
Lemma 4. [8, Lemma. 4] Let $\mathrm{A}$ be the generator of a strongly continuous cosine operator function $(C(t))_{t \in \mathbb{R}}$ Let $A$ be the generator of the cosine operator function $C$. For $t \in \mathbb{R}$ and $\lambda \in \mathbb{C}$, let

$$
S_{\lambda}(t) x:=\int_{0}^{t} \sinh \lambda(t-s) C(s) x d s, \quad x \in X .
$$

Then $S_{\lambda}(t) \in \mathcal{B}(X)$ is an operator that commutes with $A$, and

$$
\left(A-\lambda^{2}\right) S_{\lambda}(t) x=\lambda(C(t)-\cosh \lambda t) x
$$

for all $x \in X$.

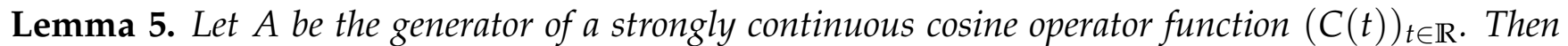
for all $t \neq 0$ and $\lambda \in \mathbb{C}$ with $\lambda t \notin i \pi \mathbb{Z}$, there exist two operators $L_{\lambda}(t), G_{\lambda}(t) \in \mathcal{B}(X)$ such that

$$
\left(A-\lambda^{2}\right) L_{\lambda}(t)+G_{\lambda}(t) S_{\lambda}(t)=I .
$$

Moreover, the operators $L_{\lambda}(t), S_{\lambda}(t), G_{\lambda}(t)$ and $A-\lambda^{2}$ are mutually commuting.

Proof. For all $t \neq 0$ and $\lambda \in \mathbb{C}$ with $\lambda t \notin i \pi \mathbb{Z}$, let $K_{\lambda}(t) x:=\int_{0}^{t} \sinh \lambda(t-s) S_{\lambda}(s) x d s$. It is clear that $K_{\lambda}(t)$ is a bounded linear operator on $X$. We consider the function $f: s \in[0, t] \longrightarrow$ $\sinh \lambda(t-s) S_{\lambda}(s) x$, then $f$ is Bochner integrable and $f(s) \in D(A)$ for all $s \in[0, t]$. Moreover, $\left(A-\lambda^{2}\right) f(s)=\lambda \sinh \lambda(t-s)(C(t)-(\cosh \lambda t) I)$ is Bochner integrable. From [1, Proposition 1.1.7], $K_{\lambda}(t) x \in D(A)$ and

$$
\begin{aligned}
\left(A-\lambda^{2}\right) K_{\lambda}(t) x & =\int_{0}^{t} \lambda \sinh \lambda(t-s)(C(s)-\cosh \lambda s) x d s \\
& =\lambda \int_{0}^{t} \lambda \sinh \lambda(t-s) C(s) x d s-\lambda \int_{0}^{t} \sinh \lambda(t-s) \cosh \lambda s x d s \\
& =\lambda S_{\lambda}(t) x-t \lambda \sinh \lambda t x .
\end{aligned}
$$

We put $L_{\lambda}(t):=(-t \lambda \sinh \lambda t)^{-1} K_{\lambda}(t)$ and $G_{\lambda}(t):=(t \sinh \lambda t)^{-1} I$. Then we have $(A-$ $\left.\lambda^{2}\right) L_{\lambda}(t)+G_{\lambda}(t) S_{\lambda}(t)=I$. Furthermore, the operators $L_{\lambda}(t), S_{\lambda}(t), G_{\lambda}(t)$ and $A-\lambda^{2}$ are mutually commuting. Indeed, since $C(s) C(t)=C(t) C(s)$ for all $s, t \geq 0$, then $S_{\lambda}(s) C(t)=$ $C(t) S_{\lambda}(s)$. Hence $S_{\lambda}(s) S_{\lambda}(t)=S_{\lambda}(t) S_{\lambda}(s)$. Thus, we deduce that $L_{\lambda}(s) S_{\lambda}(t)=S_{\lambda}(t) L_{\lambda}(s)$. On the other hand, for all $x \in D(A)$, we have

$$
\begin{aligned}
K_{\lambda}(t)\left(A-\lambda^{2}\right) x & =\int_{0}^{t} \sinh \lambda(t-s) S_{\lambda}(s)\left(A-\lambda^{2}\right) x \\
& =\lambda \int_{0}^{t} \sinh \lambda(t-s)(C(s)-(\cosh \lambda s) I) x d s \\
& =\lambda S_{\lambda}(t) x-t \lambda \sinh \lambda t x \\
& =\left(A-\lambda^{2}\right) K_{\lambda}(t) x .
\end{aligned}
$$

Then, $L_{\lambda}(t)$ commute with each one of $S_{\lambda}(t), G_{\lambda}(t)$ and $\left(A-\lambda^{2}\right)$. 
Lemma 6. Let $A$ be the generator of a strongly continuous cosine operator function $(C(t))_{t \in \mathbb{R}}$. Then for all $n \in \mathbb{N}^{*}, t \neq 0$ and $\lambda \in \mathbb{C}$ with $\lambda t \notin i \pi \mathbb{Z}$, there exist two operators $F_{\lambda, n}(t), H_{\lambda, n}(t) \in \mathcal{B}(X)$ such that,

$$
\left(A-\lambda^{2}\right)^{n} F_{\lambda, n}(t)+H_{\lambda, n}(t) S_{\lambda}^{n}(t)=I .
$$

Moreover, the operators $F_{\lambda, n}(t), H_{\lambda, n}(t), S_{\lambda}^{n}(t)$ and $\left(A-\lambda^{2}\right)^{n}$ are mutually commuting.

Proof. By lemma 5 , there exist two operators $L_{\lambda}(t), G_{\lambda}(t) \in \mathcal{B}(X)$ such that

$$
\left(A-\lambda^{2}\right) L_{\lambda}(t)+G_{\lambda}(t) S_{\lambda}(t)=I .
$$

For all $n \geq 1$ and $x \in X$, we have $L_{\lambda}^{n}(t) x \in D\left(A^{n}\right)$. In fact, the proof is by induction. For $n=1$, from lemma $5 L_{\lambda}(t) x \in D(A)$. Suppose that $L_{\lambda}^{n-1}(t) x \in D\left(A^{n-1}\right)$, so $L_{\lambda}^{n}(t) x \in D\left(A^{n-1}\right)$ and

$$
\begin{aligned}
\left(A-\lambda^{2}\right)^{n-1} L_{\lambda}^{n}(t) x & =\left[\left(A-\lambda^{2}\right) L_{\lambda}(t)\right]^{n-1} L_{\lambda}(t) x \\
& =L_{\lambda}(t)\left[\left(A-\lambda^{2}\right) L_{\lambda}(t)\right]^{n-1} x \in D(A),
\end{aligned}
$$

hence, $L_{\lambda}^{n}(t) x \in D\left(A^{n}\right)$. Furthermore,

$$
\begin{aligned}
\left(A-\lambda^{2}\right)^{n} L_{\lambda}^{n}(t) & =\left[\left(A-\lambda^{2}\right) L_{\lambda}(t)\right]^{n} \\
& =\left[I-G_{\lambda}(t) S_{\lambda}(t)\right]^{n} \\
& =I-T_{\lambda, n}(t) S_{\lambda}(t),
\end{aligned}
$$

with $T_{\lambda, n}(t)=\sum_{k=1}^{n}(-1)^{k-1}\left(\begin{array}{l}n \\ k\end{array}\right) G_{\lambda}^{k}(t) S_{\lambda}^{k-1}(t)$. So $\left(A-\lambda^{2}\right)^{n} L_{\lambda}^{n}(t)+T_{\lambda, n} S_{\lambda}(t)=I$. Similarly, we have

$$
\begin{aligned}
T_{\lambda, n}^{n}(t) S_{\lambda}^{n}(t) & =\left[I-\left(A-\lambda^{2}\right)^{n} L_{\lambda}^{n}(t)\right]^{n} \\
& =I-\left(A-\lambda^{2}\right)^{n} \sum_{k=1}^{n}(-1)^{k-1}\left(\begin{array}{l}
n \\
k
\end{array}\right)\left(A-\lambda^{2}\right)^{n(k-1)} L_{\lambda}^{n k}(t) .
\end{aligned}
$$

We define $\left.F_{\lambda, n} t\right)=\sum_{k=1}^{n}(-1)^{k-1}\left(\begin{array}{l}n \\ k\end{array}\right)\left(A-\lambda^{2}\right)^{n(k-1)} L_{\lambda}^{n k}(t)$ and $H_{\lambda, n}(t)=T_{\lambda, n}^{n}(t)$. Then $(A-$ $\left.\lambda^{2}\right)^{n} F_{\lambda, n}(t)+H_{\lambda, n}(t) S_{\lambda}^{n}(t)=I$. Moreover the operators $\left(A-\lambda^{2}\right)^{n}, F_{\lambda, n}(t), H_{\lambda, n}(t)$ and $S_{\lambda}^{n}(t)$ are pairwise commuting.

Lemma 7. Let $A$ be the generator of a strongly continuous cosine operator function $(C(t))_{t \in \mathbb{R} \text {. Then }}$ for all $q \in \mathbb{N}, t \neq 0$ and $\lambda \in \mathbb{C}$ with $\lambda t \notin i \pi \mathbb{Z}$, if $\mathcal{R}(C(t)-\cosh \lambda t)^{q}$ is closed, then $\mathcal{R}\left(A-\lambda^{2}\right)^{q}$ is also closed.

Proof. Let $\left(y_{n}\right)_{n \in \mathbb{N}}$ be a sequence in $\mathcal{R}\left(A-\lambda^{2}\right)^{q}$ converging to $y \in X$. Then there is a sequence $\left(x_{n}\right)_{n \in \mathbb{N}}$ of $\mathcal{D}\left(A^{q}\right)$ satisfying $\left(A-\lambda^{2}\right)^{q} x_{n}=y_{n}$. By Lemma 6, we obtain

$$
\left(A-\lambda^{2}\right)^{q} F_{\lambda, q}(t) y_{n}+H_{\lambda, q}(t) S_{\lambda}^{q}(t) y_{n}=y_{n} .
$$

Hence, we conclude that

$$
\lambda^{q}(C(t)-\cosh \lambda t)^{q} H_{\lambda, q}^{q}(t) x_{n}=S_{\lambda}^{q}(t)\left(A-\lambda^{2}\right)^{q} H_{\lambda, q}^{q}(t) x_{n}
$$




$$
\begin{aligned}
& =H_{\lambda, q}^{q}(t) S_{\lambda}^{q}(t)\left(A-\lambda^{2}\right)^{q} x_{n} \\
& =H_{\lambda, q}^{q}(t) S_{\lambda}^{q}(t) y_{n} \\
& =y_{n}-\left(A-\lambda^{2}\right)^{q} F_{\lambda, q}(t) y_{n} .
\end{aligned}
$$

Thus, $y_{n}-\left(A-\lambda^{2}\right)^{q} F_{\lambda, q}(t) y_{n} \in \mathcal{R}(C(t)-\cosh \lambda t)^{q}$. Since $\mathcal{R}(C(t)-\cosh \lambda t)^{q}$ is closed and $\left(A-\lambda^{2}\right)^{q} F_{\lambda, q}(t)$ a bounded linear operator, it follows that the sequence $y_{n}-\left(A-\lambda^{2}\right)^{q} F_{\lambda, q}(t) y_{n}$ converges to $y-\left(A-\lambda^{2}\right)^{q} F_{\lambda, q}(t) y$ as $n$ tends to $\infty$ and

$$
y-\left(A-\lambda^{2}\right)^{q} F_{\lambda, q}(t) y \in \mathcal{R}(C(t)-\cosh \lambda t)^{q} \subseteq \mathcal{R}\left(A-\lambda^{2}\right)^{q} .
$$

We obtain $y \in \mathcal{R}\left(A-\lambda^{2}\right)^{q}$, which completes the proof.

Corollary 8. Let $(C(t))_{t \in \mathbb{R}}$ be a strongly continuous cosine operator function on $X$ with generator $A$. Then, if $C(t)-\cosh \lambda t$ is semi-regular operator and $\lambda t \notin i \pi \mathbb{Z}$, then $A-\lambda^{2}$ is also semi-regular operator.

Proof. If $C(t)-\cosh \lambda t$ is semi-regular operator, then $\mathcal{R}(C(t)-\cosh \lambda t)$ is closed and $\mathcal{N}(C(t)-$ $\cosh \lambda t) \subseteq \mathcal{R}^{\infty}(C(t)-\cosh \lambda t)$. By lemma $7, \mathcal{R}\left(A-\lambda^{2}\right)$ is closed and $N\left(A-\lambda^{2}\right) \subseteq N(C(t)-$ $\cosh \lambda t) \subseteq \mathcal{R}^{\infty}(C(t)-\cosh \lambda t) \subseteq \mathcal{R}^{\infty}\left(A-\lambda^{2}\right)$. Hence $A-\lambda^{2}$ is semi-regular operator.

(1) Assume that $C(t)-\cosh \lambda t$ is a Saphar operator and $\lambda t \notin i \pi \mathbb{Z}$, then $C(t)-\cosh \lambda t$ has a generalized inverse and $C(t)-\cosh \lambda t$ is semi-regular. By corollary $8 C(t)-\cosh \lambda t$ is semi-regular. It remains to prove that $A-\lambda^{2}$ has a generalized inverse. Since $C(t)-$ $\cosh \lambda t$ has a generalized inverse, then by [7, Proposition 1] there exists $R \in \mathcal{B}(X)$ such that,

$$
(C(t)-\cosh \lambda t) R(C(t)-\cosh \lambda t)=C(t)-\cosh \lambda t .
$$

According to lemma 5, we have $\left(A-\lambda^{2}\right) L_{\lambda}(t)+G_{\lambda}(t) S_{\lambda}(t)=I$, then

$$
\begin{aligned}
\left(A-\lambda^{2}\right) & =\left(A-\lambda^{2}\right) L_{\lambda}(t)\left(A-\lambda^{2}\right)+S_{\lambda}(t) G_{\lambda}(t)\left(A-\lambda^{2}\right) \\
& =\left(A-\lambda^{2}\right) L_{\lambda}(t)\left(A-\lambda^{2}\right)+(C(t)-\cosh \lambda t) G_{\lambda}(t) \\
& =\left(A-\lambda^{2}\right) L_{\lambda}(t)\left(A-\lambda^{2}\right)+(C(t)-\cosh \lambda t) R(C(t)-\cosh \lambda t) G_{\lambda}(t) \\
& =\left(A-\lambda^{2}\right) L_{\lambda}(t)\left(A-\lambda^{2}\right)+\left(A-\lambda^{2}\right) S_{\lambda}(t) R\left(A-\lambda^{2}\right) S_{\lambda}(t) G_{\lambda}(t) \\
& =\left(A-\lambda^{2}\right) L_{\lambda}(t)\left(A-\lambda^{2}\right)+\left(A-\lambda^{2}\right) S_{\lambda}(t) R S_{\lambda}(t) G_{\lambda}(t)\left(A-\lambda^{2}\right) \\
& =\left(A-\lambda^{2}\right)\left[L_{\lambda}(t)+S_{\lambda}(t) R S_{\lambda}(t) G_{\lambda}(t)\right]\left(A-\lambda^{2}\right) .
\end{aligned}
$$

Let $H_{\lambda}=L_{\lambda}(t)+S_{\lambda}(t) R S_{\lambda}(t) G_{\lambda}(t)$. Then $H_{\lambda}$ is a bounded linear operator, $R\left(H_{\lambda}\right) \subseteq$ $\mathcal{D}(A)$ and $\left(A-\lambda^{2}\right) H_{\lambda}\left(A-\lambda^{2}\right)=A-\lambda^{2}$. Let $K_{\lambda}=H_{\lambda}\left(A-\lambda^{2}\right) H_{\lambda}$, it follows that $K_{\lambda}$ and $R\left(K_{\lambda}\right) \subseteq \mathcal{D}(A)$. Moreover, we have

$$
\begin{aligned}
\left(A-\lambda^{2}\right) K_{\lambda}\left(A-\lambda^{2}\right) & =\left(A-\lambda^{2}\right) H_{\lambda}\left(A-\lambda^{2}\right) H_{\lambda}\left(A-\lambda^{2}\right), \\
& =\left(A-\lambda^{2}\right) H_{\lambda}\left(A-\lambda^{2}\right),
\end{aligned}
$$




$$
=A-\lambda^{2}
$$

and

$$
\begin{aligned}
K_{\lambda}\left(A-\lambda^{2}\right) K_{\lambda} & =H_{\lambda}\left(A-\lambda^{2}\right) H_{\lambda}\left(A-\lambda^{2}\right) H_{\lambda} \\
& =H_{\lambda}\left(A-\lambda^{2}\right) H_{\lambda} \\
& =K_{\lambda}
\end{aligned}
$$

Hence $A-\lambda^{2}$ has a generalized inverse. Consequently $A-\lambda^{2}$ is a Saphar operator.

(2) Assume that $C(t)-\cosh \lambda t$ is quasi-Fredholm. First, we prove the $\operatorname{dis}\left(A-\lambda^{2}\right)$ is finite. Since $\operatorname{dis}(C(t)-\cosh \lambda t)$ is finite, then there exists $d \in \mathbb{N}$ for all $n \geq d$,

$R(C(t)-\cosh \lambda t)^{n} \cap \mathcal{N}(C(t)-\cosh \lambda t)=\mathcal{R}(C(t)-\cosh \lambda t)^{d} \cap \mathcal{N}(C(t)-\cosh \lambda t)$.

We show that for all $n \geq d$,

$$
\mathcal{R}\left(A-\lambda^{2}\right)^{n} \cap \mathcal{N}\left(A-\lambda^{2}\right)=\mathcal{R}\left(A-\lambda^{2}\right)^{d} \cap \mathcal{N}\left(A-\lambda^{2}\right) .
$$

Let $y \in \mathcal{R}\left(A-\lambda^{2}\right)^{d} \cap \mathcal{N}\left(A-\lambda^{2}\right)$, then there exists $x \in \mathcal{D}\left(A^{d}\right)$ such that $y=\left(A-\lambda^{2}\right)^{d} x$. Then, according to lemma 5 , there exist two operators $F_{\lambda, d}(t)$ and $H_{\lambda, d}(t)$ such that

$$
\left(A-\lambda^{2}\right)^{d} F_{\lambda, d}(t)+S_{\lambda}^{d}(t) H_{\lambda, d}(t)=I .
$$

Therefore,

$$
\begin{aligned}
y & =\left(A-\lambda^{2}\right)^{d} F_{\lambda, d}(t) y+S_{\lambda}^{d}(t) H_{\lambda, d}(t) y, \\
& =F_{\lambda, d}(t)\left(A-\lambda^{2}\right)^{d} y+(C(t)-\cosh \lambda t)^{d} H_{\lambda, d}(t) x, \\
& =(C(t)-\cosh \lambda t)^{d} H_{\lambda, d}(t) x .
\end{aligned}
$$

Therefore,

$y \in \mathcal{R}(C(t)-\cosh \lambda t)^{d} \cap \mathcal{N}(C(t)-\cosh \lambda t) \subseteq \mathcal{R}(C(t)-\cosh \lambda t)^{n} \subseteq \mathcal{R}(\lambda-A)^{n}$.

Consequently $y \in \mathcal{R}(\lambda-A)^{n} \cap \mathcal{N}\left(A-\lambda^{2}\right)$. Hence $\operatorname{dis}\left(A-\lambda^{2}\right)$ is finite.

Next we show that $\mathcal{R}\left(A-\lambda^{2}\right)+\mathcal{N}\left(A-\lambda^{2}\right)^{n}$ is closed in $X$, for all $n \geq d$. If $n=0$, then from lemma $7, \mathcal{R}\left(A-\lambda^{2}\right)$ is closed. If $n \geq$ let $y_{p}=\left(A-\lambda^{2}\right) x_{p}+z_{p}$ be a sequence which converges to $y(p \rightarrow \infty)$, with $x_{p} \in X$ and $z_{p} \in \mathcal{N}\left(A-\lambda^{2}\right)^{n}$. As $S_{\lambda}^{n}(t) y_{p}=$ $S_{\lambda}^{n}(t)\left(A-\lambda^{2}\right) x_{p}+S_{\lambda}^{n}(t) z_{p} \in \mathcal{R}(C(t)-\cosh \lambda t)+\mathcal{N}(C(t)-\cosh \lambda t)^{n}$, then $S_{\lambda}^{n}(t) y \in$ $\mathcal{R}(C(t)-\cosh \lambda t)+\mathcal{N}(C(t)-\cosh \lambda t)^{n}$. There exist $x \in X$ and $z \in \mathcal{N}(C(t)-\cosh \lambda t)^{n}$ such that $S_{\lambda}^{n}(t) y=(C(t)-\cosh \lambda t) x+z$. So $S_{\lambda}^{2 n}(t) y=S_{\lambda}^{n}(t)(C(t)-\cosh \lambda t) x+S_{\lambda}^{n}(t) z$ where $S_{\lambda}^{n}(t) z \in \mathcal{N}\left(A-\lambda^{2}\right)^{n}$, which implies that,

$$
\begin{aligned}
y & =\left(A-\lambda^{2}\right)^{n} F_{\lambda, n}(t) y+S_{\lambda}^{2 n}(t) H_{\lambda, n}(t) y, \\
& =\left(A-\lambda^{2}\right)^{n} F_{\lambda, n}(t) y+H_{\lambda, n}(t) S_{\lambda}^{n}(t)(C(t)-\cosh \lambda t) x+H_{\lambda, n}(t) S_{\lambda}^{n}(t) z, \\
& =\left(\left(A-\lambda^{2}\right)^{n} F_{\lambda, n}(t) y+H_{\lambda, n}(t) S_{\lambda}^{n}(t)\right)+H_{\lambda, n}(t) S_{\lambda}^{n}(t) z .
\end{aligned}
$$

Therefore, $y \in \mathcal{R}\left(A-\lambda^{2}\right)+\mathcal{N}\left(A-\lambda^{2}\right)^{n}$. Hence $\mathcal{R}\left(A-\lambda^{2}\right)+\mathcal{N}\left(A-\lambda^{2}\right)^{n}$ is closed. 
Proposition 9. Let $(C(t))_{t \in \mathbb{R}}$ be a strongly continuous cosine operator function on $X$ with generator A satisfying $\|C(t)\|=\circ\left(t^{2}\right)(t \rightarrow \infty)$. The following assertions are equivalent:

(1) $A$ is bounded below,

(2) $A$ is semi-regular.

Proof. $(1) \Rightarrow(2)$. Obvious.

$(2) \Rightarrow(1)$. Let $y \in \mathcal{N}(A)$. Since $A$ is semi-regular, then $\mathcal{N}(A) \subseteq \mathcal{R}(A)$ and $\mathcal{R}(A)$ is closed.

So there exists $x \in \mathcal{D}(A)$ such that $y=A x$. From [8, Theorem.7], $C(t) x=x+\frac{t^{2}}{2 !} A x+$ $\int_{0}^{t} \frac{(t-s)^{3}}{3 !} C(s) A^{2} x d s$, for all $t \neq 0$. As $\|C(t)\|=o\left(t^{2}\right)$, then $y=0$. Which implies that $A$ is bounded below.

\section{Acknowledgments}

I am very grateful to the reviewer for the careful reading of the paper and the valuable suggestions and constructive comments which helped to enrich the content and improve the presentation of the result in this paper.

\section{References}

[1] W. Arendt, C. J. K. Batty, M. Hieber, and F. Neubrander, Vector-valued Laplace Transforms and Cauchy Problems, Monographs in Mathematics, 96, Birkhäuser-Springer, Basel, 2011.

[2] H. O. Fattorini, Ordinary differential equations in linear topological space, II, J. Differential Equations 6 (1969), 50-70.

[3] J.P. Labrousse, Inverses généralisés d'opérateurs non-bornés, Proc. Amer. Math. Soc. 115(1992), 125-129.

[4] J.P. LAbrousse, Les opérateurs quasi-Fredholm: une généralisation des opérateurs semi-Fredholm, Rend. Circ. Math. Palermo (2), XXIX (1980) 161-258.

[5] D. Lutz, Strongly continuous operator cosine functions, Functional Analysis (Dubrovnik, 1981). (Lecture Notes in Math., Vol. 948), Springer, Berlin-New York, 1982, 73-97.

[6] M. Мвекнта, A. OuAнав, Opérateur s-régulier dans un espace de Banach et théorie spectrale, Acta Sci. Math. (Szeged) 59 (1994), 525-543.

[7] V. MüLlen, Spectral Theory of Linear Operators and Spectral Systems in Banach Algebras, 2nd edition. Oper. Theory Advances and Applications, vol. 139 (2007).

[8] B. Nagy, On cosine operator functions on Banach spaces, Acta Sci. Math. Szeged 36 (1974), 281-290.

[9] A. Tajmouati, M. Амоuchв, M. Karmounia Symmetric Difference Between Pseudo B-Fredholm Spectrum and Spectra Originated from Fredholm Theory, Filomat 31(16)(2017), 5057-5064.

[10] C. C. Travis and G. F. Webb, Cosine families and abstract nonlinear second order differential equations, Acta Math. Acad. Sci. Hungar. 32 (1978),75-96.

[11] H. O. FATTORINI, Uniformly bounded cosine functions in Hilbert spaces, Indiana Univ. Math. J. 20 (1970), 411-425.

[12] S. Kurepa, cosine functkmal equation in Hilbert space, Canad. J. Math., 12 (1960), 45-49. 